\title{
Smart Home Monitoring using Android and Wireless Sensors
}

\author{
Suresh Sankaranarayanan ${ }^{a^{*}}$, Au Thien Wan ${ }^{b}$, Aida Harayani Pusa ${ }^{c}$ \\ ${ }^{a, b}$ Institut Teknologi Brunei, Jalan Tungku Link, Gadong, Brunei Darussalam \\ ${ }^{c}$ Infomars Training and Technology Solutions, Berakas, Brunei Darussalam
}

\begin{abstract}
Common electrical hazards that occur at home are usually associated with the usage of faulty home appliances and electrical distribution. Circuit breakers are normally used to protect overloading at residential homes. We envisage that by monitoring continuously the usage characteristics of electrical power at every power point in real time could be the best method for protection. Presently, wireless sensor network (WSN) has been used in home monitoring to optimally manage the 'well-being' of power distribution in home in order to minimize hazards caused by electrical faults. This technique incidentally helps controlling the tariff by maintaining the power usage optimally. It has also been found that not much study has yet been made towards the use of WSN for monitoring electrical power and the consequent hazards with a view to ensure the welfare of residents at home. Based on this idea, we have developed a real time WSN based Electrical Hazard monitoring system that also uses android mobile application environment. The android based smart home monitoring, christened as ABASH system the details of which we present in this paper, detects the threshold violation in electrical parameters and produces an appropriate alert signal for home owners to enable them to take proper remedial actions depending on the situation, much before the circuit breaker operates. The proposed system has been validated specifically for the Brunei Electric standard as a case study. The details are provided in the paper.
\end{abstract}

\section{Index Terms: WSN, ABASH.}

(C) 2014 Published by MECS Publisher. Selection and/or peer review under responsibility of the Research Association of Modern Education and Computer Science.

\section{Introduction}

Electrical safety and warnings have always been taken for granted especially during the utilization of home appliances. So people are not aware that without carrying out a safety inspection based on the visible indication, there could be a possibility of fault getting initiated which would result in producing an electrical hazard such as fire. The electrical energy that flows from home distribution board (DB) is consumed by home appliances through their respective switch inlets when they are switched ON. During the course of operation any abnormal electrical condition can occur due to certain fault conditions, for example; overloaded circuit of the appliance, and damaged insulation, misuse of extension cords and electrical products.

\footnotetext{
*Suresh Sankaranarayanan. Tel: 6732461020

E-mail Address: suresh.sn@itb.edu.bn
} 
In a residential home, a distribution board normally takes care of any electrical fault that occurs. However, its functionality is such that it cannot completely distinguish certain problem that is dangerous to us. One tends to call a qualified electrician or licensed electrical inspector to do a safety inspection and repair at homes [1].

The inspection is usually done by looking at electrical power conditions, for example, checking for correct wattage of light bulbs or installing a Ground Fault Circuit Interpreter (GFCI) or determining the power connectivity between socket outlets using multimeter. Occurrences of electrical hazards are difficult to predict and to take notice because, most of the characteristics of electrical faults lies in the electrical wiring [2]. Such electrical hazards that a house tenant tends to overlook are overheating, unusual smell, short circuits, sparks and sputters [2]. Over a period time, this lack of awareness may lead to devastating effects such as fire and so forth.

We will now consider the use of Wireless Sensor Networks (WSN) for this purpose. In several industrial applications, WSN is being profusely used; for example in environmental monitoring, traffic control, weather checking, monitoring of temperature, etc. Countless amount of research work being carried out on smart home monitoring, are usually associated by implementing intelligent agent with WSN for controlling appliances or monitoring elders who stayed at home alone [3], etc. In one of the research work in vogue towards smart home monitoring [4], intelligent device-level residential energy monitoring and controlling platform has been developed that is based on interactive outlets and light dimmer-switches. Such a platform allows users to monitor, control and compare electricity consumption of appliances connected to the residential power network. In this prototype it is shown as possible to perform control via remote control, mobile phone, TV or web interface. The system intelligently controls power consumption of all appliances connected to the smart network, also contributing to energy savings in the household.

In another research towards smart home monitoring, an automated power management system is shown as capable of reducing energy wastes while preserving the perceived comfort level. With this aim viz., a Green Building, a sensor-based intelligent system [5] has been implemented to monitor the energy consumption and to automatically control the behavior of appliances used in the building. Green Building concept has been implemented as a prototype and has been experimented in a real household scenario also. The analysis of the experimental results highlights that Green Building is able to provide significant energy savings also.

It may also be mentioned here that research has been carried out [6] involving WSN for electrical fault monitoring. It is used to identify phase-to-phase short circuit and single phase to earth short circuit, and it is implemented in the incoming and outgoing power line of secondary substation. This research raised a theory of monitoring loads, to locate short circuit current and single phase to earth based on WSN to determine the fault occurrence So a survey of the above mentioned works, it has become clear that there has been only a limited amount of research that has been carried out using WSN application for electrical fault monitoring at homes. In general it has been noticed that research concentration has been more only on electrical usage/consumption [4] [5].

In the research paper [6] the concept of developing a new device that would help detecting a certain electrical problem and present a solution to reduce the ignition of fire before it happens, has been introduced. This device has a specialized built in wireless sensor technology. This seems to be a much better solution compared to the existing ones, wherein the circuit breaker trips once an electrical fault happens and at times results in fire also.

With the above in view, in this research study we have presented the complete details of a unique system called ABASH - an Android BAsed Smart Home monitoring application, integrated with advance technology of wireless sensors for monitoring electrical hazards in a residential environment, periodically. An introductory detail on this system has been presented elsewhere [7]. The rest of this paper is organized as follows. Section 2 gives a brief survey of research on related technologies. Section 3 talks on ABASH - our proposed system architecture and algorithm utilized. Section 4 gives details on the implementation of the proposed system. Section 5 gives the concluding remarks.

\subsection{Research Survey}




\subsubsection{Traditional Electrical Protection}

In addition to what we have already said in the introduction section, we will now be discussing in this section more related research work that is being carried out before presenting the details about our smart home monitoring application, integrated with advance technology of wireless sensors for monitoring electrical hazards in a residential environment, periodically.

In domestic installations [8], overload protection and short-circuit protection are both provided by the same device: either a fuse or Miniature Circuit Breaker (MCB). Whether a fuse or MCB is used, only when the current exceeds a certain limit for a certain time, the fuse will break or the MCB will trip. A fuse is a simple device that limits the current flowing in an electrical circuit; it is a basic method of over-current protection that is placed inside a plug to get fused when the threshold current level is exceeded.

Distribution board is the main electrical system in a home which distributes the incoming electrical energy to power up various electrical appliances in the home through appropriate socket outlets and switches. The distribution board consists of MCB and Earth Leakage Circuit Breaker (ELCB), to protect us from hazards that can cause a common electrical fault such as short circuit and ground fault [8].

Nevertheless, as a problem occurs during the distribution such as wiring failure, weary metal casing inside outlet or faulty appliance and others, it results in spark or rather short-circuit. This repeated action could initiate fire because the board keeps on distributing incoming current. The process may soon cause heat and melting of the insulation of the socket outlet which later can cause fire. According to WiseGEEK [9], "Electrical power can also cause heat energy, and this excessive heat can cause the insulation around the wires to melt away". Once an electrical wire becomes exposed, it can come into contact with the metal casing of the outlet or even with the other circuit wire. If a connection then gets made, electrons leap across the gap and form a visible spark. Since the connection shortens the intended distance of the circuit, it is frequently called a short or short circuit. A short circuit caused by overheated wiring not only can lead an outlet to start sparking, it can also create the ignition source for a dangerous electrical fire.

One study [8] mentions "Fires can be caused by electrical system failures, appliance defects, incorrectly installed wiring, misuse and poor maintenance of electrical appliances, and overloaded circuits and extension cords". These electrical faults, if left unattended can cause loss of property and loss of life. Thus, from the above study it can be inferred that the existing systems do not provide enough protection for tenants and their property because they do not provide enough indication for alerting them when hazard would happen. To have an electrician to inspect and to check regularly the electrical conditions of a house may not be cost effective and practical.

\subsubsection{WSN for Electrical Hazard Monitoring}

WSN in terms of networking is a spatially distributed autonomous sensor devices employed to cooperatively monitor the physical or environmental conditions or parameters at different locations in real time. Every sensor node is equipped with a transducer/sensor, microcomputer, transceiver and a power source, say a Mote [9] like device. Such a device then generates electrical signals based on physical effects and phenomena sensed. The microcomputer in the device processes and stores the sensor output. Once the transceiver receives a command from a central computer, it then transmits data to that computer which is a system for measuring parameter threshold.

Common parameters to be sensed by such WSN devices while monitoring are temperature, humidity, pressure, wind direction and speed, illumination intensity, vibration intensity, sound intensity, power-line voltage, chemical concentrations, pollutant levels and vital body functions [10]. Many projects can therefore be performed mostly on recognizing what causes fault on home power line [11].

In one another research [6] WSN is used for electrical fault monitoring towards detecting and locating electrical system faults including phase-phase short circuit and single phase to earth short. In this scheme, a sensor measures only the phase current characteristics of the wire that is attached to it, but not synchronized to 
other sensors. However, the validity of analysis depends on the measurement accuracy, especially, in the small amplitude of fault current system of connecting to ground with compensation coil. The accuracy that can be achieved depends on the components that are used in the sensor design and the sampling algorithm. The reliability and stability of the fault detection and location based on WSN shall however, be tested in practice.

In another study WSN is used towards monitoring the power and its control for electrical home appliance to manipulate the power consumption of appliances [12]. In this research an embedded remote electric power monitor/control System is used which is very easy to install and does not need additional wiring. This system enables the user to control the on/off and the power consumption remotely without bothering with extra wiring. Thus the system is very convenient and can be used at once after just plugging it in. The embedded system and the key components for home power management have been developed, and in order to utilize remote control, the Power Line Communication (PLC) technologies for power management have been integrated. The proposed PLC Power controlled Outlet Module (PPCOM) is designed for remote monitoring and controlling of different electric home appliances connected over a PLC network in a home environment. The embedded home server connects to the Internet by the Asymmetric Digital Subscriber Line (ADSL). Thus the user can manage electric home appliances and remotely monitor their power consumption status by means of a remote Web browser.

Other researchers have been applying wireless sensors towards monitoring electrical consumption usage [4] [5]. Eco-System for Smart Home (ESH) [4] successfully monitors the home for different events that are usually overlooked, such as children watching TV instead of doing their homework or the oven left on after leaving the house. Alongside with the feedback on current energy consumption status obtained from a number of outlets, consumers are able to set a list of actions for each change in the current energy consumption scheme. Users become aware of residential energy consumption by receiving notifications on mobile phones, personal web-page profiles or TV sets.

The main contribution of ESH is in possibility to execute user defined behavioral patterns, which connects various residential sensors with power consumption of household appliances with intention to improve the energy efficiency and safety. The safety means that every plugged device is monitored. If the consumption crosses the thresholds, an outlet will immediately switch off the power. Each of outlets has safety priority this means that, e.g. an oven has one of the highest priorities, and if no person is detected in a house, but consumption on the oven is detected, the smart outlet can immediately switch off the power, or notify a user to manually switch it off remotely (via web interface). That way we would make the installation easier and more ubiquitous at the same time improving the comfort of living and efficiency in energy consumption.

In another research, on Green Building [5] a system for efficient electrical-energy management in residential and business buildings has been suggested. The goal of Green Building is twofold. From one hand, it allows the user to better understand the energy consumption of each single appliance, thus bringing him/her to make more intelligent choices in terms of energy consumptions. On the other hand, it allows improved energy efficiency by means of an appropriate management of each single appliance, depending on rules specified by the user. Green Building was implemented as a prototype system and deployed it in a real household scenario. The experimental results show that Green Building is able to provide significant energy savings, by eliminating standby consumptions and/or adapting the behavior of appliances to the real environmental conditions. The main objection to using a tool like Green Building is that its cost may be too high compared to the economic saving it can provide. Actually, current costs of electricity sensors and actuators are very high. However, they are expected to decrease as soon as their penetration in the market increases. In addition, next-generation electrical appliances will include built-in sensors and actuators. General Electric has already developed a complete suite of smart appliances using ZigBee [14] for wireless communication among them and/or with a central server

Use of WSN technology has also inspired the proposed system viz., ABASH[7] because not much study are available on handling electrical hazard parameter from both usage of appliances and electrical power distribution except for the one being developed by EFCI [13]. 
EFCI [13] has introduced their special built in plug and EFCI receptacle that is specially designed for providing wattage and sensor parameter for measuring conditions. Both items are used to identify the type of appliances that are plugged into EFCI receptacle and send information to their system and integrate with the values captured from sensor for calculating fault and its consumption. With their system, it can be used to monitor the fluctuation of the electrical flow and control the usage of power by automatically manipulating its switch. The requirement of the research is to have both plug and receptacle in order to monitor the progress from electrical faults. This is for effective measurement between sensors. Fig. 1 shows the EFCI Safe plug receptacle. Fig. 2 shows implementation of EFCI Safe plug receptacle in home socket outlet.

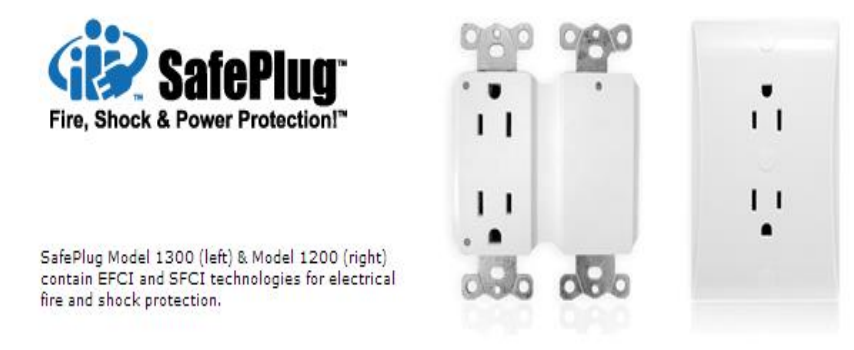

Fig.1 Safe Plug Receptacles

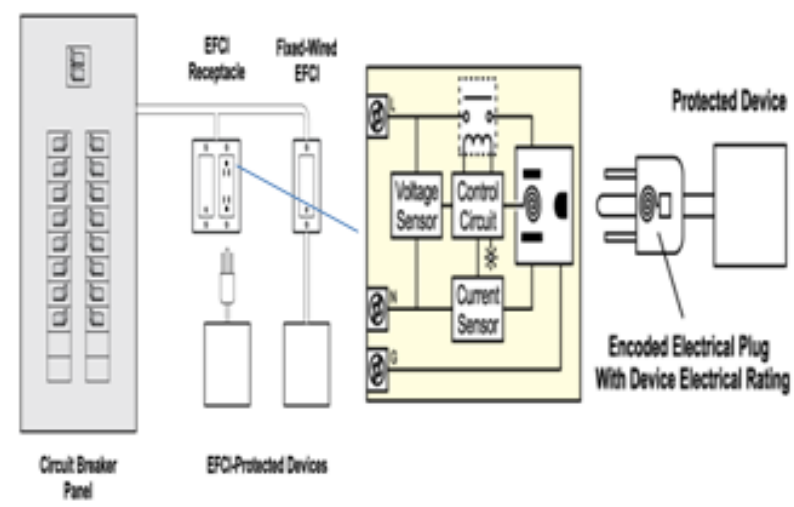

Fig.2 Safe Plug receptacle in Home socket

In none of the above research has there been any work reported on using WSN towards continuous monitoring electrical hazard parameters in residential homes for taking preemptive preventive action before any actual devastating fault happens. In a similar research carried out where EFCI [13] has developed a special built-in plug with sensor for capturing value for detecting fault and its consumption of power once appliance is plugged in. But we need to monitor the electrical parameters continuously towards fault diagnosis beforehand and take action. Here we have developed ABASH [7] which uses the theory of EFCI [13] for placing sensors onto socket outlet to capture each of the parameter values periodically towards fault monitoring and to recommend actions appropriately. This is one of the significant aims to measure electrical abnormalities during the utilization of home appliances. In ABASH development; in addition a temperature sensor is used to perform temperature measurements for threshold violation unlike in the Safe Plug. The reason behind the addition is because of Brunei's climate changes, temperature ranges from $24^{\circ} \mathrm{C}$ to $33^{\circ} \mathrm{C}$. ABASH system will 
use sensors to monitor temperature, voltage and current specifically to determine the anomalies in electrical distribution during the usage of appliances through the common socket outlets. As the threshold is detected during the process, it will produce an alert from the system to intimate the tenants for appropriate action. With the ability of ABASH, it would be possible to create an awareness for the owner to take preventive or remedial action before the actual hazard occurs. With the advancement of science and technology, WSN usage for monitoring has enhanced online access with several types of platform which allows integration of wireless sensor technology. Soon, the innovations in smart home monitoring will increase and the vision of smart building will be envisioned.

\section{ABASH - Android Based Smart Home Monitoring}

Having seen the motivation towards developing ABASH [7], the system architecture that has been followed as shown in Fig.3, represents the system structures, its components, the properties of its components and the relationships (e.g. the behavior) between them.

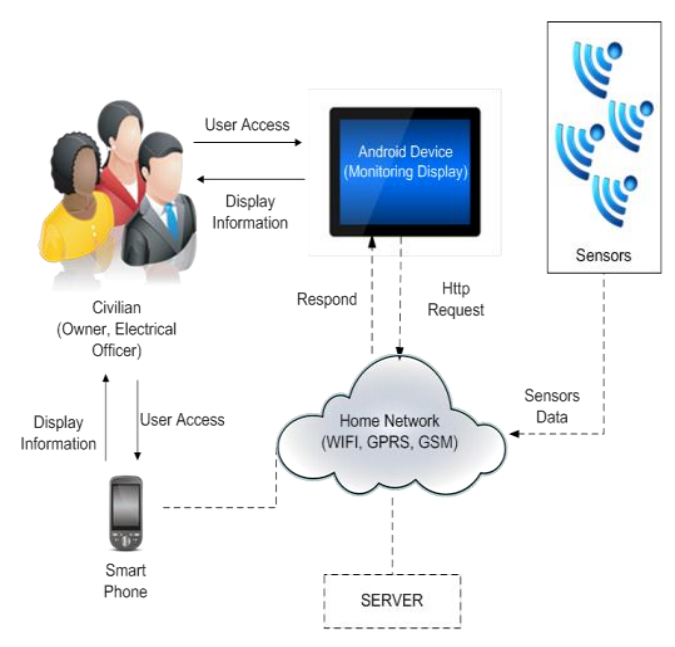

Fig.3 ABASH System Architecture

The system architecture consists of the following components:

- Civilian - tenant/main user

- Android device - interface application

- Sensors - wireless sensor device

- Server - the storage

- Wireless network for home - main connectivity

When the user tries to access the system for information they will use the android device as an interface between the system and the sensor in order to interact and retrieve data. This application is used for displaying information that captured by the sensors. The wireless sensor which is implemented for each room socket outlet will start to process as soon as the user requests for the sensor information. Upon the request, the sensor will respond and deliver the information through a home network and save the data on the server which later is used for displaying as requested by the user. When using a smart phone, the functions will be similar to an android application as explained above. 
As we use sensors in our system at socket outlets for monitoring electrical parameters, it is important to see as to which routing protocol fits in well with the WSN used. The routing protocols are classified into one of the ensuing three models: Single hop model, Multi-hop model and Cluster-based hierarchical model [14][15]. In ABASH, cluster based model as shown in Fig.4 has been found to be suitable, because it involves laying out the sensors according the outlet of each room. In the Cluster-based model, the network is divided into clusters, room wise, comprising of a number of nodes. Cluster head, which is a master node, within each respective cluster is responsible for routing the information from its constituent sensors to other cluster heads [14].

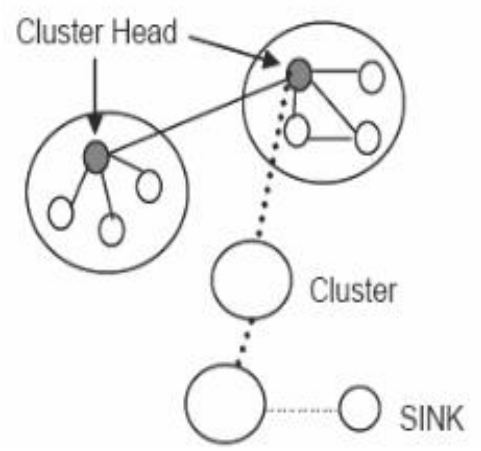

Fig.4 Cluster based model

ABASH architecture has similar elements of sensor node and the master node as mentioned in one of the research paper [14][15]. The current and voltage sensors are chosen because they produce data that is useful for measuring threshold of a certain point of fault in electrical distribution. The sensors measure the values of voltage, current and temperature by an appliance to determine if it is on/off or above the threshold level and communicate this information to a master node when polled. ABASH will contact the master node which controls the polling of the sensors, and provides GUI to the monitor, and log sensors events. Fig.5 shows typical Sensor Layout.

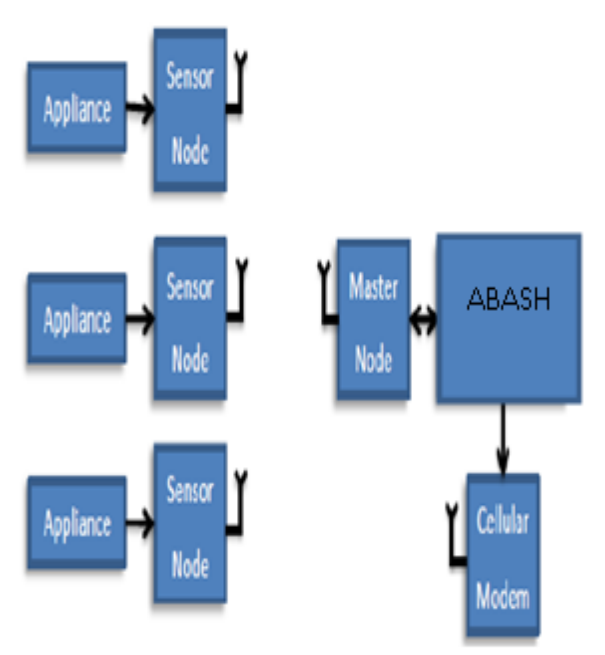

Fig.5 Typical sensor layout 


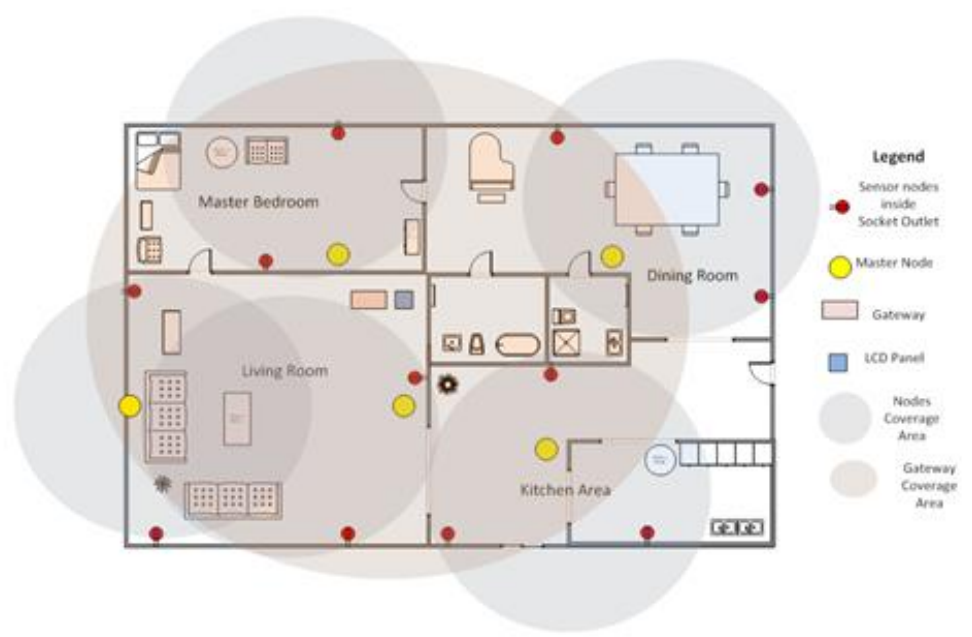

Fig.6 Sensor implementation for different rooms

Fig.4 is the design of the infrastructure using the studies from researchers [16][17]. Each sensor node will be placed accordingly at all socket outlets inside each room. The nearest master node in gateway coverage will act automatically as the main sensor to collect and distribute information from the sensor nodes as soon as it is requested by the user using the system LCD panel or handheld android device. The master node will respond by collecting and organizing the information which are then transmitted through a network gateway and back to the system for data display. The sensor will start to process as the user clicks start button in the system to initiate information retrieval for parameters of the socket under monitoring. Fig. 6 shows sensor implementation for different rooms in house. In the system features, the design is to provide flexible monitoring so it will able to read the incoming data smartly. This system features are:

- Read incoming data from specialized socket

- Detect electrical abnormalities through socket activity

- Display event graphically

- Alert by notifying occupants about the threshold violation

- Allow configuration for room and device

The application of android device as an interface to the system will allow the system to provide information display to the user. Not only that, it will also act as an interaction between specialized devices to display its information as a system is initiated. Before initiating the process, any new device allocated in a new room will need to be registered to the system to allow the device to function fully. Then as the process starts with a click of a button, the system will cooperate with the nearest specialized device and request them to start collecting and organized the information which then be transmitted to the system. The transmission is required in order to process the real time information for identifying electrical abnormalities. Any information that recognized as a threshold violation will be notified onto the device screen by changing the color values

The device is considered as a specialized socket because it is built with three types of sensor that are used for detecting anomalies in electrical flow from the socket outlet at home. The concept is adapted from Safeplug [1] where it uses only two parameters such as current and voltage for measuring incoming data from the distribution board. The difference to this specialized socket is that, there is additional sensor to measure temperature which is important to be implemented due to Brunei climate condition and it also follows the standard used by Brunei Electrical Distribution. This specialized socket contains three types of built-in sensors 
such as Temperature, Voltage and Current. It then provides real time data from three sensors and placed in each room of the house for socket data retrieval. The built in sensor for the specialized device is for detecting three different parameters in real time as it is applied sparsely in different room. It is combined as one in the specialized socket to make the socket capable to work fully so it's able to produce exact threshold violation for alerting the user. This is done by notifying through the system. The advantage for having this socket outlet is to allow user to implement them physically onto socket outlet without any configuration or wiring to it.

With the system architecture of ABASH as shown in Fig. 3, the functionalities i.e. House Agent which is ABASH LCD panel or Android, Sensor Agent and Database Agent, are illustrated below in the algorithm used:

\section{House Agent}

- $\quad$ Start GUI to get Sensor Agent parameters

- Input Occupant mobile number for receiving alert SMS

- Input Room for number of socket outlet

- Input type of parameters agent into room

- Input Upper and lower range of temperature, current and voltage

- Start Sensor Agent (initiation)

- When a request is activated based on parameters, check Device setting for Sensor Agent

- When Sensor Agent is found send request to the sensor agent for current, voltage and temperature data

- Suspend behavior and wait a reply from Sensor Agent

- When temperature, current, voltage reading is received, an update parameter table with temperature, current, voltage reading and time are recorded.

- When there are 5 consecutive parameters reading out from normal parameter range, trigger an alert to Occupant

- Reschedule requests for temperature, current and voltage based on given parameter

- Create graphs to simulate parameter values according to time

\section{Sensor Agent}

- Initiating the Sensor Agent by the house agent

- Register the sensor Agent in Device Setting

- Get the current, temperature, voltage value from the rooms

- Suspend the sensor agent until the next initiate request for each parameter value

\section{Database Agent}

- Register sensor to device to use its services to advertise

- Suspend agent until database entries are received from House Agent

- When the parameter's value is received, extract data and store in database

- Reschedule the request for database entries to be updated

\section{ABASH Implementation using Android}

The implementation of ABASH involved several languages and software. The languages used to suit the development of the interface are HTML, JavaScript, PHP and MySQL. For simulating Wireless Sensor part towards capturing Voltage, Current and Temperature, Java Script was used to produce their values randomly in accordance to real time situation. The reason for using JavaScript is due to its ability to combine with Jquerymobile interface. PHP is used with MySQL database engine for data storing. 
The main objective of the system is to provide information captured by the device to monitor values that are set by using socket setting and use the information to detect any values that are considered as threshold violation. Two buttons are important in this process; one for requesting sensor or device to perform their activity as well as to automatically measure performance and other to stop the process. In this case their activity is to verify for incoming value from the socket and deliver to the main system for measurement. These are shown in Fig.7.

In ABASH when there is a new room built at home, the detail need to be registered in the system to make a relationship for device to allocate its incoming data. The room table will be used as a link with device id. Fig. 8 shows when new room is created e.g. Bed Room A with total socket of 2. Fig.9 shows the listing of rooms with sockets

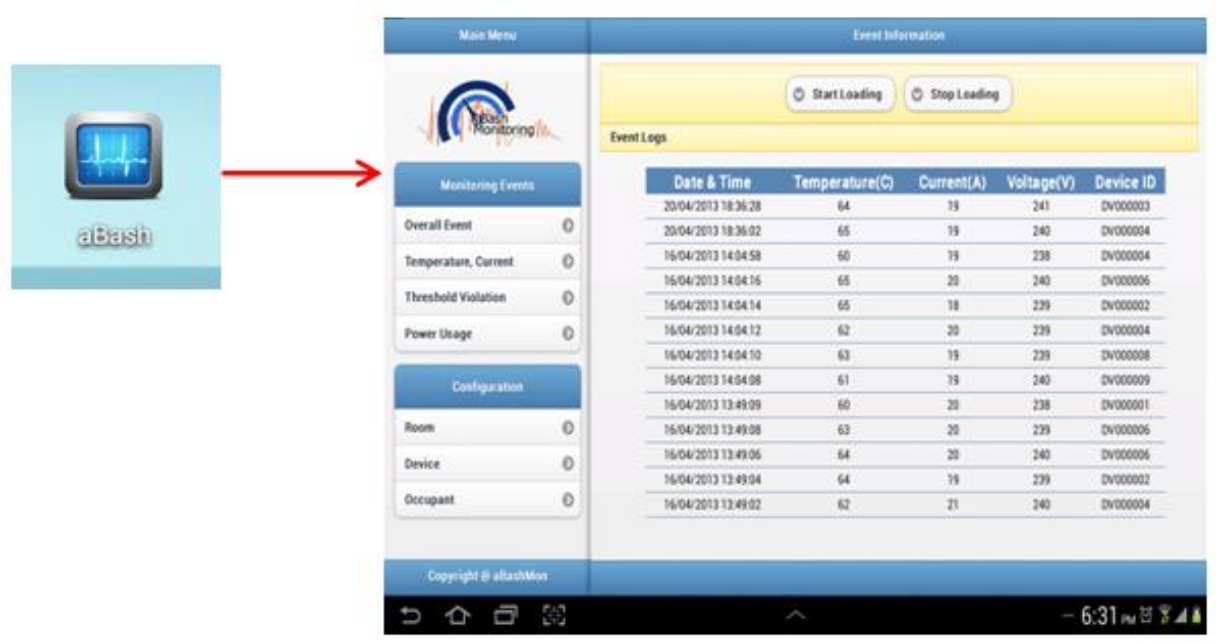

Fig.7 ABASH User Interface

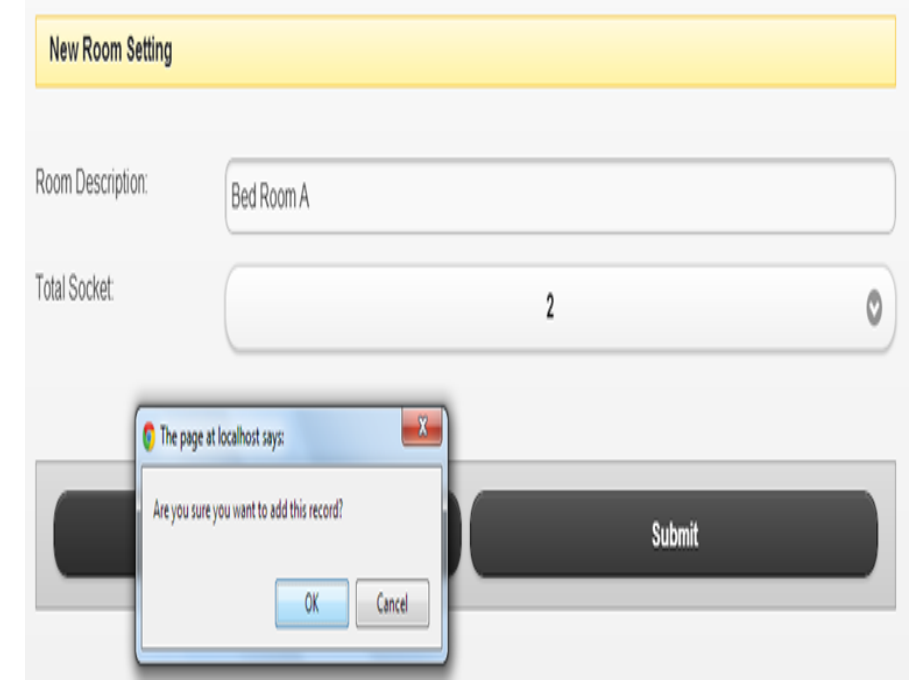

Fig.8 New Room Setting 


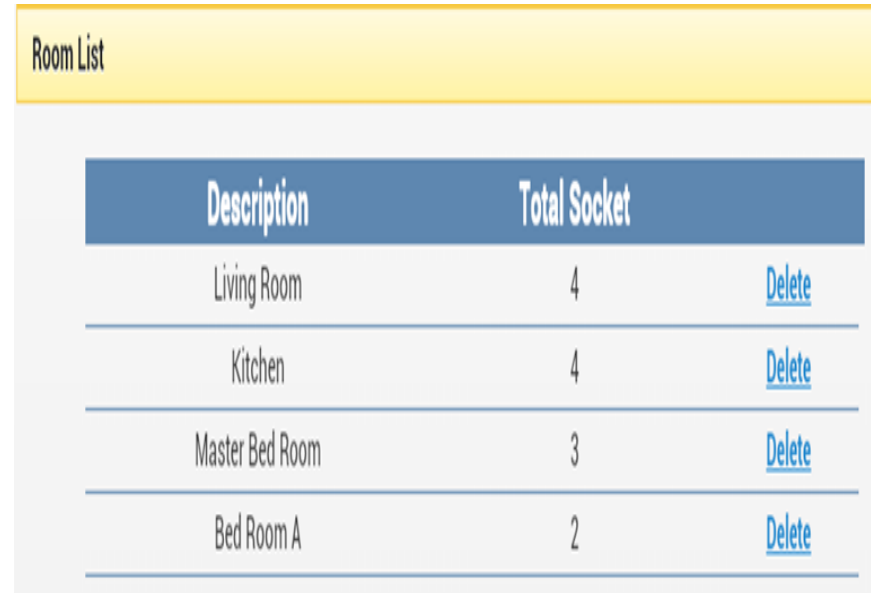

Fig.9 Room Listing with socket

Another important method after setting up new room is configuration for socket outlet. This configuration is for setting a condition for specialized device because it is a requirement for different type of appliance used. When registering a device, it is required to set the conditions of socket outlet for detecting threshold violations during real time data polling. The reason for not setting the value similarly for all sockets is because each appliance at home uses different type of amp rating (current value) to utilities. It's the main core of ABASH monitoring so it is capable to notify users at the point of fault occurrence. Fig.10 shows the socket outlet setting and Fig.11 shows the new configuration setting list.

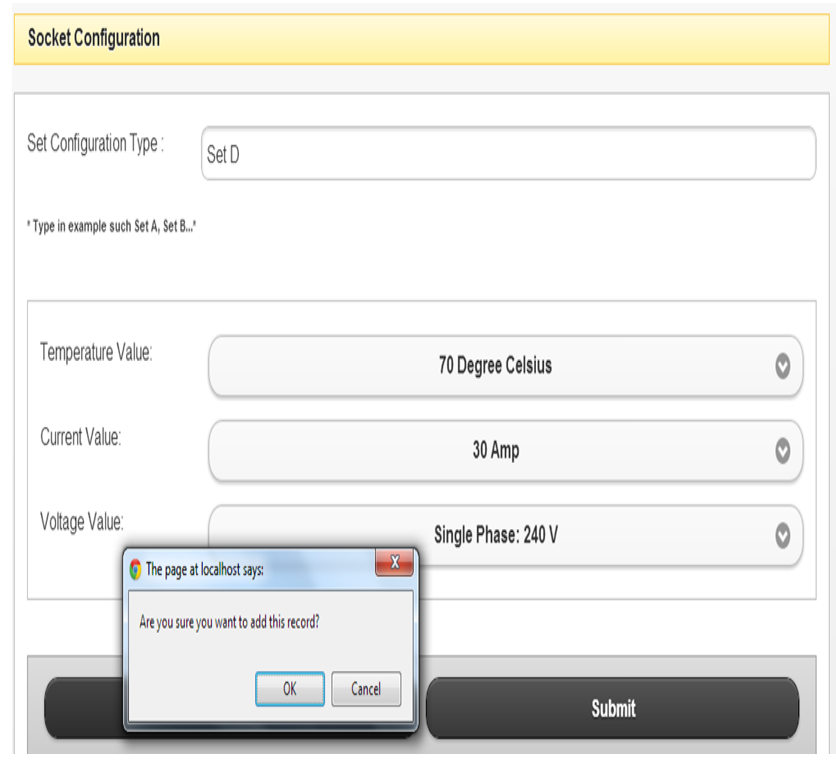

Fig.10 Socket Outlet Settings 


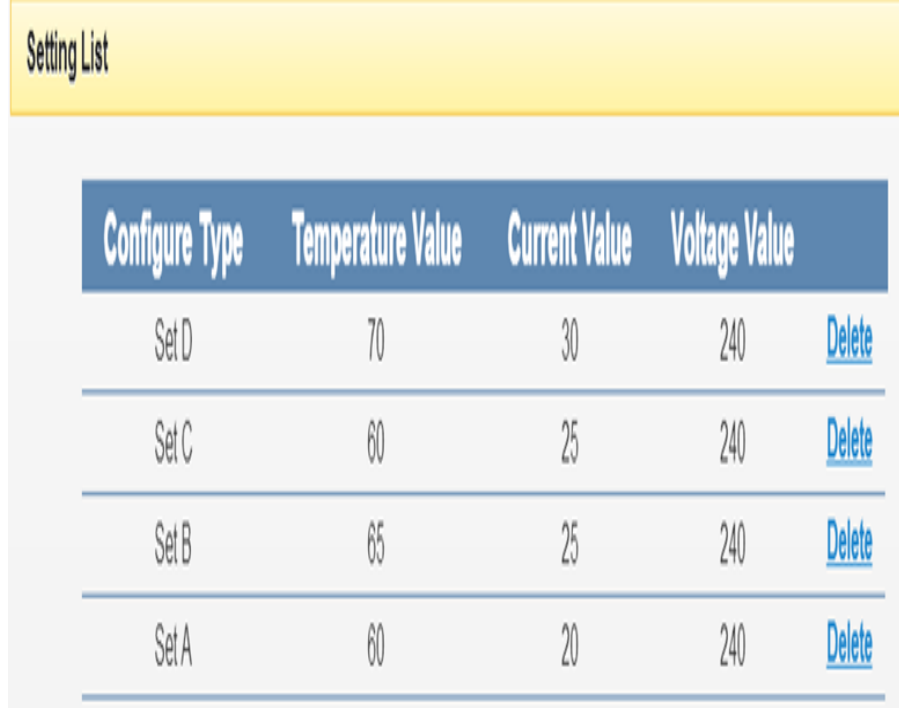

Fig.11 New Configuration Setting List

The significant part of the monitoring process is the registration of room device. Without this registration there will be no projection of values for each parameter (current, temperature and voltage). This is required by the system to evaluate measurement of threshold violation. During the process of inputting values for each socket, inside the device form will display the list of configurations suitable for the socket outlet in the room. When Socket Setting is clicked from device form as shown in Fig.12, it will list the value that was saved from the socket setting table as shown in Fig.10. Fig.13 shows the socket setting for new device

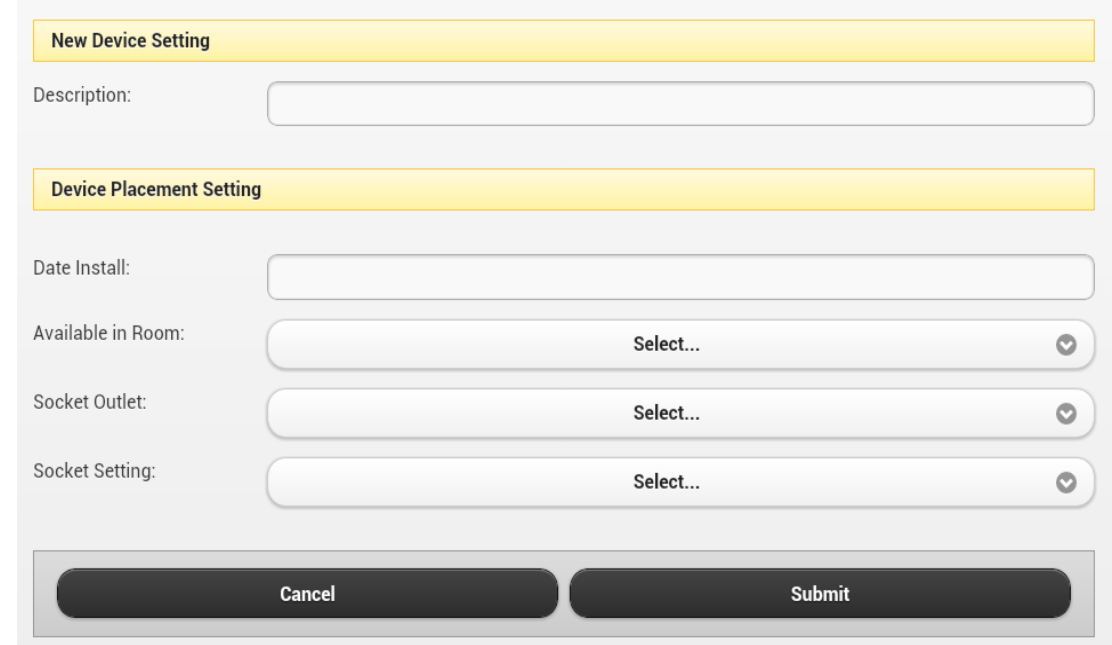

Fig.12 New Device Setting Form 


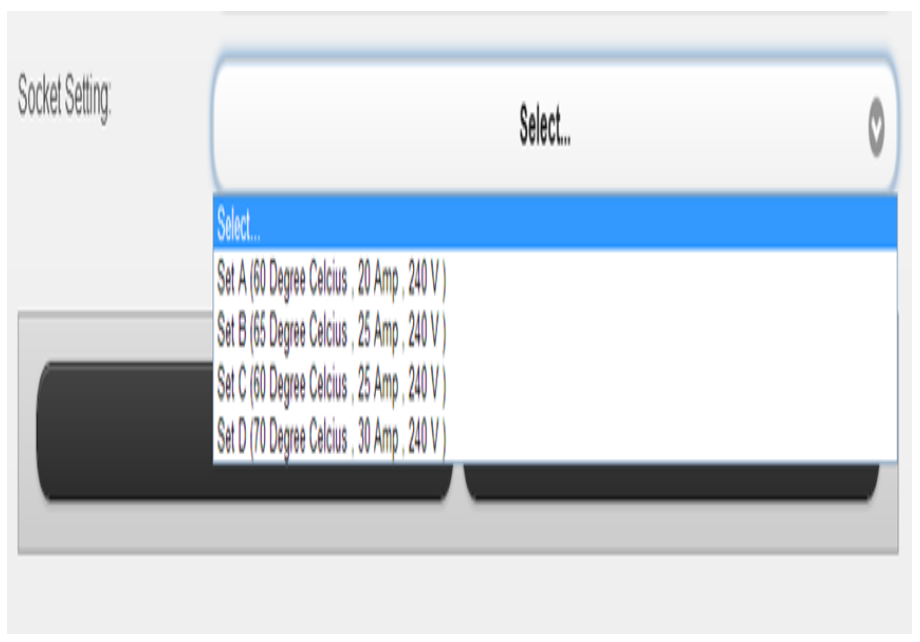

Fig.13 Socket setting for Device

Every new device that is implemented for home must be registered in system to provide a relationship between the registered rooms. This is essential because it will be easier for users to identify the whereabouts of problem device at home. Fig.9 show data of registered room. For Example, Bed room A contain two sockets which mean the registered device will be two set of values e.g. DV000006 and DV000005. Fig.14 and 15 shows the device registered into system and also listing of registered device for registered room.

Fig.16 also shows Event log being recorded which is an important monitoring display for ABASH system for users to read. It displays real time values resulting from electrical appliance usage anytime captured by registered device. This device function is to collect parameter values from each sensor built within the socket outlet during the operation of the appliances. The main objective of event log monitoring is for intimating user when the monitored parameters exceed the threshold value.

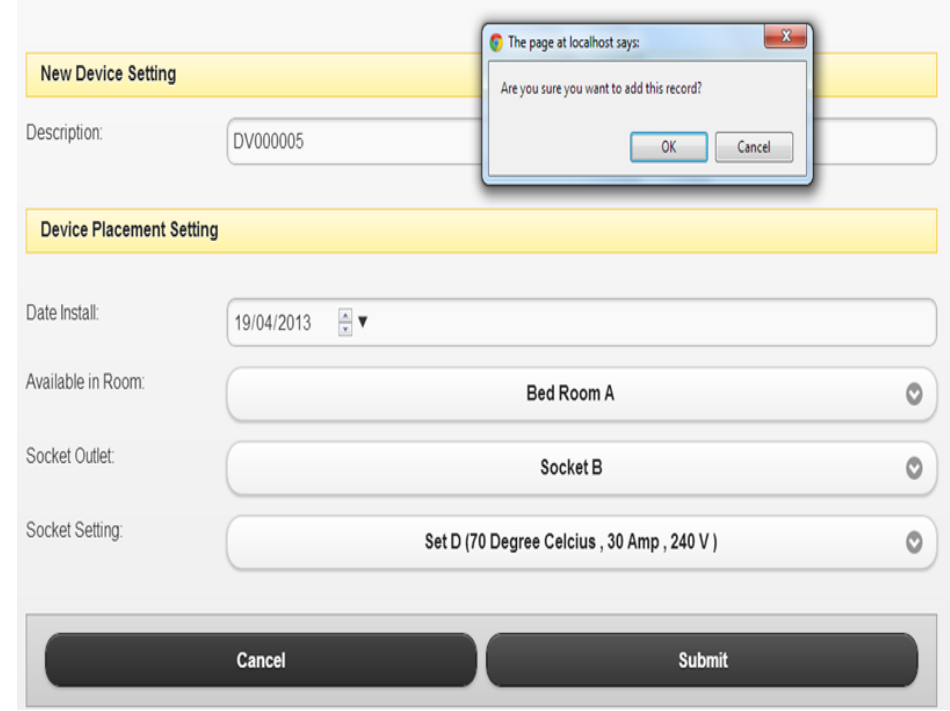

Fig.14 DV000005 Registered 


\begin{tabular}{|c|c|c|c|c|c|}
\hline \multicolumn{6}{|l|}{ Device Lists } \\
\hline Description & Date & Room & Sodket Outlet & Socket Sotting & \\
\hline DV000009 & $010.03: 2013$ & Master Bed Room & SocketC & SetA & Delete \\
\hline DV000008 & $22 \cdot 02 \cdot 2013$ & Master Bed Room & Socket B & SetA & Deletet \\
\hline Dv000007 & 06.02 .2013 & Master Bed Room & SocketA & SetA & Delete \\
\hline DV000000 & $19 \cdot 02.2013$ & BedroomA & Socket B & SetD & Delete \\
\hline Dvo00005 & $10.03 \cdot 2013$ & BedRoomA & SocketA & SetA & Deleter \\
\hline DV000004 & 03.02 .2013 & Living Room & SocketD & SetA & $\underline{\text { Delete }}$ \\
\hline Droocoos & $04.02: 2013$ & Living Room & SocketC & SetA & $\underline{\text { Delete }}$ \\
\hline DV000002 & 08.012 .2013 & LimingRoom & SocketB & SetA & Delete \\
\hline Dvocooou & $03.01 \cdot 2013$ & Living Room & SocketA & SetA & Delete \\
\hline
\end{tabular}

Fig.15 Listing of Registered Device

As an example in showing threshold violation, Bedroom A (Socket A) of device id number DV000005 shows the occurrence of threshold violation and it notifies the system by making them in red color. Therefore, to identify whether to take an action or not is by learning how many times the system detected the violations (marked in red) for a certain device. Repetitive warning of threshold of similar device means it requires an action by the user. This is shown in Fig.17. It is seen from Fig. 17 that Device ID DV000005 in Bed room A has repetitive threshold violation i.e。 5 times, which is shown in red. This is an indication to user to take appropriate action. In addition this threshold violation values can also be referred in threshold violation event in the system where it captures all the red value from event log for user reference.

\begin{tabular}{|c|c|c|c|c|}
\hline Threshold Violation Logs & & & & \\
\hline Date \& Time & Temperature(C) & Current(A) & Voltage(V) & Device [D \\
\hline $07 / 05 / 201316 \cdot 44: 48$ & 64 & 19 & 240 & DV000008 \\
\hline 07/05/2013 16:44:32 & 62 & 19 & 240 & DV000005 \\
\hline $07 / 05: 201316.38 .44$ & 62 & 20 & 241 & DV000005 \\
\hline $07 / 05 / 201316.34: 01$ & 62 & 18 & 239 & DV000009 \\
\hline $07 / 05 / 201316: 30-12$ & 62 & 20 & 240 & DV000007 \\
\hline $07 / 05 / 201316.29 \cdot 41$ & 62 & 19 & 240 & DV000003 \\
\hline $07 / 05 / 201316.25 .22$ & 63 & 20 & 240 & DV000009 \\
\hline $07 / 05 / 201316 \cdot 25.13$ & 62 & 20 & 241 & DV000009 \\
\hline $05 / 05 / 201316: 24: 30$ & 64 & 20 & 240 & DV000005 \\
\hline $0405 / 201315: 09: 13$ & 64 & 21 & 239 & DV000008 \\
\hline $0305 / 201316.36 .08$ & 64 & 19 & 240 & DV000001 \\
\hline $1504201316 \cdot 39 \cdot 27$ & 63 & 19 & 240 & DV000005 \\
\hline $13: 04201316.36 .13$ & 63 & 20 & 241 & DV000005 \\
\hline $11 / 04201310.43: 31$ & 65 & 19 & 239 & DV000005 \\
\hline $0404201315: 17: 48$ & 64 & 20 & 241 & DV000009 \\
\hline
\end{tabular}

Fig.16 Event Log Monitored 


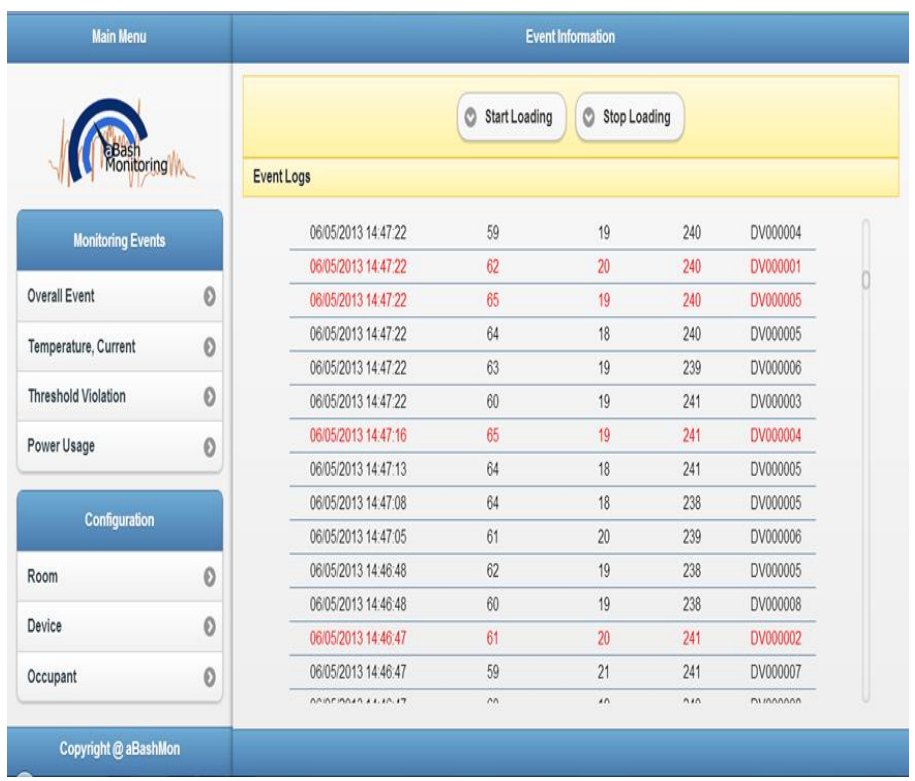

Fig.17 Repetitive threshold indication from DV000005

The captured values saved in the event can be used for displaying temperature, current as well as used for power consumption computation. Figs. 18 to 20 show the temperature and current event values displayed in real time ordered by date, time and devices. Another important function of this system is the projection of how each appliance that plugs into the socket outlet draws their power during their operations. This could be essential to the users for check and balance on the amount of electricity they spend The power event will help user to level their bill payment as they're able to estimate and predict the consumption of electrical power for each appliance at home. The computed value of power could also be displayed in real time as a bar chart to show the daily average power used by devices as shown in Figs. 21 to 22.

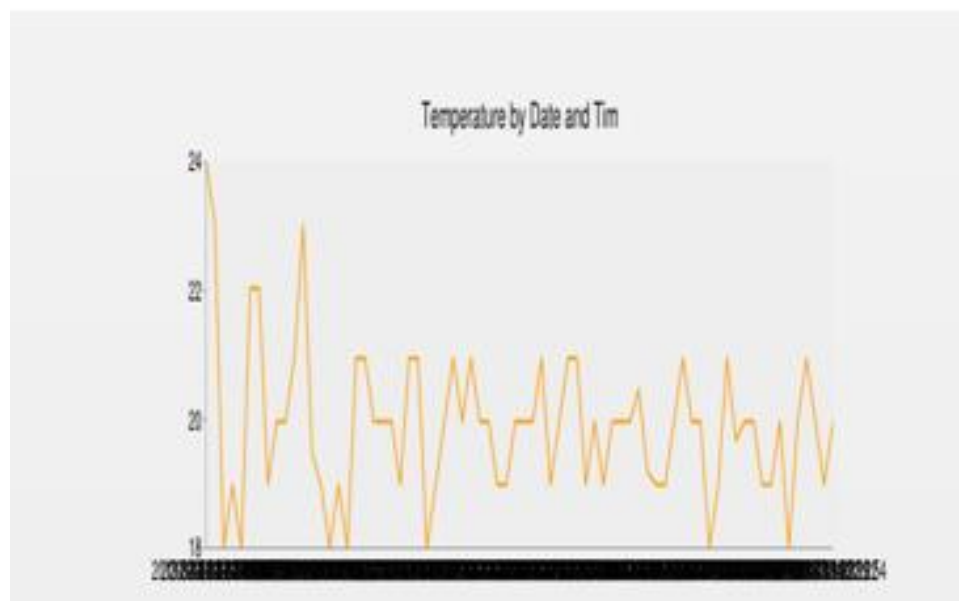

Fig.18 Temperature by Date and Time 


\section{Temperature and Curret Chart}

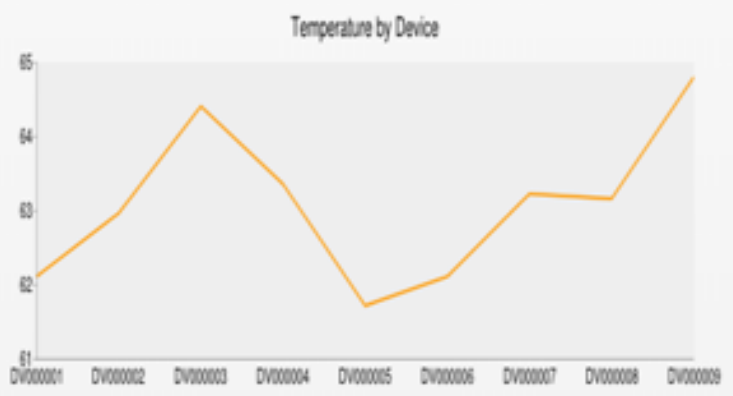

Fig.19 Temperature by Device

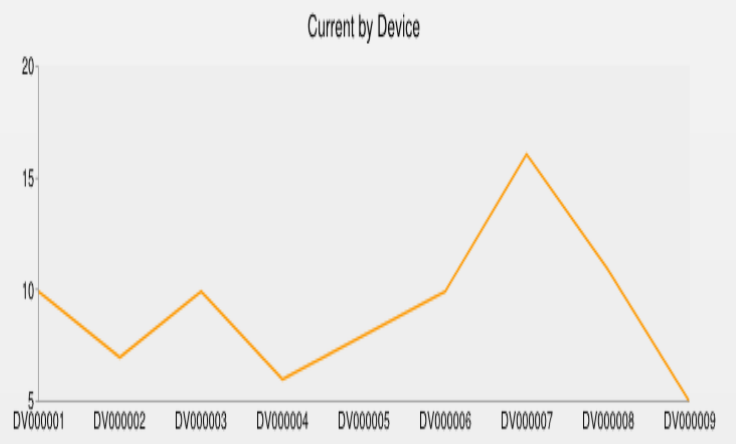

Fig.20 Current by Device

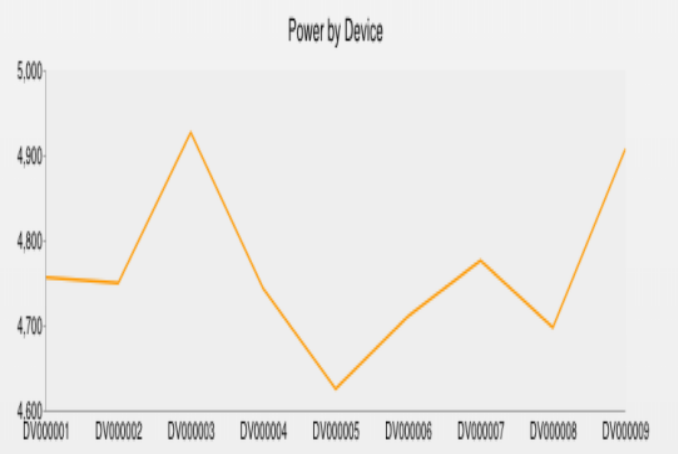

Fig.21 Power Consumption by Device 


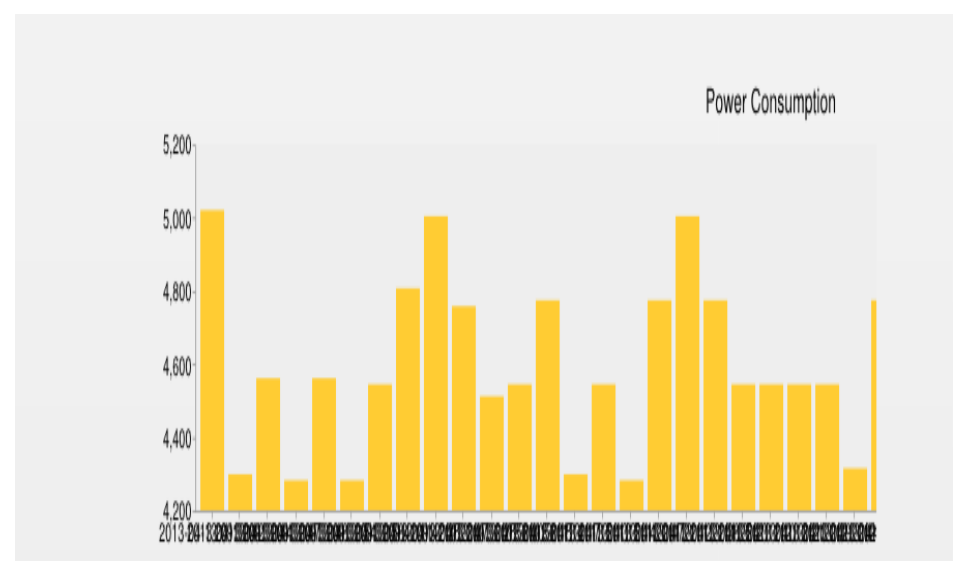

Fig.22 Power Consumption by Date and Time

\section{Conclusion \& Future Work}

Circuit breaker and MCB at home may offer initial electrical protection to home dwellers but they are not that effective sometimes. Recently research has also been carried out by installing sensor in socket outlet towards monitoring power consumption at home and also electrical fault monitoring in substation. It is seen from the literature that there has been special socket outlet developed with built in sensor for measuring current and voltage which is according to EFCI 2008. Our proposed ABASH, an android enabled smart home monitoring system, has been developed for monitoring possible faulty appliances or detect anomalies before any disastrous consequences happen to a home. The system we proposed would measure current, voltage and temperature periodically from each socket outlet of room and send to system for computing towards threshold violation and appropriate action by user before circuit breaker gets tripped or fire breakout. The application for different parameter under monitoring is chosen according to Brunei climate condition and electrical standard so it is able to predict real time threshold violation to make user to be aware of what's happening during their usage of home appliance. In addition the system also shows temperature, current and power consumption graphically to users on the ABASH system based on values recorded from each appliance. The system is also useful for check and balance on the consumption in the electricity through the power event log. The monitoring using ABASH is o suitable to be used for all types of houses in Brunei whether it is old or new. In future, an advanced graphical interface can be developed for the system where it's capable of showing and monitoring of parameters in every room. In addition to showing threshold violation in event log to user, it would be beneficial to provide audio alarm from the system towards taking immediate action and also provide SMS alert on smart phone to home users and Electrical department.

\section{References}

[1] DoItYourself Staff. Electrical Safety and Hazard FAQs. 1995, Retrieved from http://www.doityourself.com/stry/ homeelec\#b

[2] John M. Understanding Electrical Fire Safety, Fire Extinguisher. 2000, Retrieved from http://www.fireextinguisher101.com/article0705-electrical-fire-safety.html

[3] Rezwan I et al. Towards Universal Access to Home Monitoring for Assisted Living Environment. Proceedings of the 5th International on Conference Universal Access in Human-Computer Interaction. Part II: Intelligent and Ubiquitous Interaction Environments, Sandiego, California, USA, pp. 189 - 198, 2009. 
[4] Mrazovac B et al. Towards Ubiquitous Smart Outlets for Safety and Energetic Efficiency of Home Electric Appliances. Proceedings of 2011 IEEE International Conference on Consumer Electronics, Berlin, Germany, pp. 324-328, 2011.

[5] Anastasi G et al. An Intelligent System for Electrical Energy Management in Buildings. Proceedings of 2011 IEEE International Conference on Intelligent Systems Design and Applications, Cordoba, Spain, pp. 70, 2011.

[6] Zhai Z.N and Gui W.F. Research on Monitoring Power System Faults by Wireless Sensor Network, Proceedings of the third International Symposium on Electronic Commerce and Security Workshops (ISECS ‘ 10), Guangzhou, china, pp. 354 - 357, 2010.

[7] Suresh, S and Thien Wan, Au. ABASH - Android based Smart Home Monitoring using Wireless Sensors", Proceedings of IEEE International conference on Clean energy and Technology (CEAT), Langkawi, Malaysia, 2013, pp.482-487

[8] WireChief Electric. Common Causes of Home Electrical Fires. 2011, Retrieved from http://www.vancouverelectricianblog.com/common-home-electrical-fires/

[9] WiseGEEK. What Causes an Outlet to Spark? 2003, Retrieved from: http://www.wisegeek.com/whatcauses-an-outlet-to-spark.htm

[10] Wireless Sensor Networks Area. 2009, Retrieved from http://cal.postech.ac.kr/2009/wireless_sensor_networks_area.htm

[11] Shwetak N.P et al. At Flick of a switch: Detecting and Classifying Unique Electrical Events on Residential Power Line. 2007, Retrieved from http://abstract.cs.washington.edu/ shwetak/ papers/ubicomp2007_flick.pdf

[12] Chia, H L et al. (2008). Power monitoring and Control for Electric Home Appliances Based on Power Line Communication. PMTC 2008, IEEE International Instrument and Measurement Technology Conference, Victoria, Canada, 2008, pp.2179-2184

[13] Electrical Fault Circuit Interrupter (EFCI), 2008. Retrieved from http://www.2d2c.com/efci.php

[14] Akkaya, K and Younis, M. "A survey on routing protocols for wireless sensor networks." Journal of Adhoc Networks, 2005, Vol. (3), pp.325-349, Elsevier.

[15] Suresh, S. Hierarchical intelligent agent based wireless body sensor mesh networks. In ITNG '09: Proceedings of the 2009 Sixth International Conference on Information Technology: New Generations, Las Vegas, USA, 2009, IEEE Computer Society, pp. 1602-1603

[16] Sen Gupta, G and Hetherington, M. Miniaturization of Wireless Sensor Nodes for Smart Digital Home. Instrumentation and Measurement Technology Conference (I2MTC), Uruguay, 2012 IEEE International. p.2796- 2801

[17] Jolly, V and Latifi, S. Comprehensive Study of Routing Management in WSN- Part. Proceedings of the 2006 International Conference on Wireless Networks, ICWN, Las Vegas, USA, 2006, pp. 37-44.

\section{Author(s) Profile}

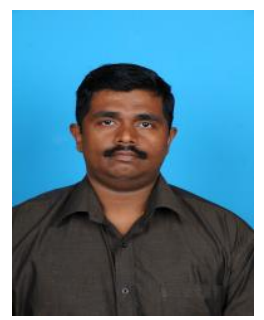

Prof. (Dr) S Suresh is an Associate Professor, Department of Computer \& Information Systems, Institute of Technology, Brunei (ITB - A technological university). He is also Senior Member of IEEE computer Society and Computer Society of India too. He has supervised more than 30 research students leading to M.Sc, ME, M.Phil and M.S degrees and currently supervising 3 students leading to M.phil and Ph.d in UWI, Jamaica and a doctoral student in Institut Teknologi Brunei respectively. He has got to his credit, as on date, more than 60 fully refereed research papers published in the Proceedings of major IEEE international conferences, as Book Chapters and in International Journals with citation of 208 and H-index of 9 . He is also a Reviewer and Technical Committee member for a number of IEEE Conferences and Journals. He has conducted many tutorials, workshops and also given Guest Lectures in 
networking in various Universities and Colleges.. He also had collaborative and funded research during 20082010 in University of WestIndies, Jamaica. He currently has a Funded project in energy where he is principal investigator. His current research interests are mainly towards 'Mobile and Ubiquitous Computing - Wireless Sensor Networks, RFID, Cloud Computing, Internet of Things, Network Congestion in wired \& wireless networks used in the Health, Commercial and Engineering sectors.

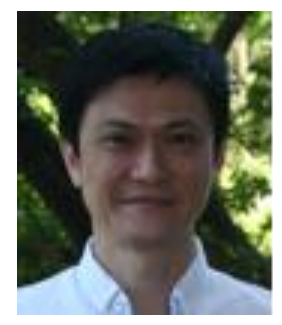

Dr. Au Thien Wan is Deputy Dean, Faculty of Business and Computing who holds Ph.d degree in Information Technology from University of Queensland, Australia in 2013. He got to his credit eight paper in reputed International conferences and Journals. His research interests are mainly in E-learning, e-health, Networking and Security. He was also awarded best paper in $23^{\text {rd }}$ Australasia conference on Information systems in 2012.

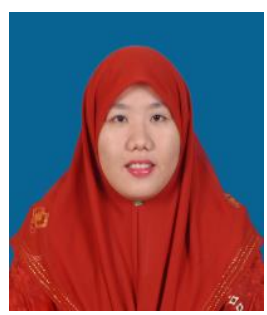

Aida Harayani Pusa is currently working in Infomars Training and Technology solutions, Brunei Darussalam. She holds Bachelor's degree (Hons) in Internet Computing from computing and Information Systems, Institut Teknologi Brunei. She possess programming skills in Java, Php, Android. Her research interests in wireless Sensors, Mobile computing, Android.

How to cite this paper: Suresh Sankaranarayanan, Au Thien Wan, Aida Harayani Pusa,"Smart Home Monitoring using Android and Wireless Sensors", IJEM, vol.4, no.2, pp.12-30, 2014.DOI: 10.5815/ijem.2014.02.02 